\title{
Dynamics of single and multiple Zener transitions
}

\author{
R. I. Cukier and M. Morillo* \\ Department of Chemistry, Michigan State University, East Lansing, Michigan 48824-1322 \\ and Center for Fundamental Materials Research, Michigan State University, East Lansing, Michigan 48824-1322
}

J. M. Casado

Fisica Teorica, Universidad de Sevilla, Apdo, Correos 1065, Sevilla 41080, Spain

(Received 5 November 1990; revised manuscript received 9 September 1991)

\begin{abstract}
The probability of remaning in a given state is evaluated for a two-level system that can cross repeatedly and periodically as a function of a control parameter. The possibility of multiple Zener transitions between the surfaces introduces the issue of the appropriate initial conditions for each successive transition. We devise a simple model, which is readily analyzed numerically, to evaluate the consequences of an initially nondiaganol density matrix on the subsequent evolution through the crossing region. The initial state strongly affects the outcome of the transition. Thus successive transitions are, in general, not independent of each other. Consequently, we find that the probability oscillates in time. This contrasts with the result of convergence at long times to a constant state occupation which would be predicted based solely on probabilities of single transitions. We also analyze the role that inelastic transitions play in modifying the nondissipative multiple-Zener-transition model. This model finds application to small tunnel junctions, where the control parameter is a current and the output is a voltage. Here, we show that the average dc voltage is nonzero when inelastic transitions are included, in contrast to the zero average voltage obtained in the absence of inelastic transitions, or when their rate is very large.
\end{abstract}

\section{INTRODUCTION}

Transitions between different potential-energy surfaces play an important role in a large variety of physical, 1,2 chemical, ${ }^{3-5}$ and biological ${ }^{6-8}$ phenomena. Typically, these transitions are promoted via a change in the system's configuration, e.g., the relative motion of molecular species entails a breakdown of the BornOppenheimer approximation and leads to a transfer of probability between potential surfaces. In other circumstances, and external perturbation will couple the surfaces. Recent examples of this latter situation are electron tunneling in very small tunnel junctions, ${ }^{9}$ where a current source is the external perturbation, and the resistance of small metal loops to a time-dependent magnetic flux. ${ }^{10,11}$ Other examples have recently been discussed by Gefen, Ben-Jacob, and Caldeira. ${ }^{12}$ These transitions are often referred to as Zener ${ }^{13-16}$ transitions, and we will maintain this terminology.

In an externally driven situation, there is the possibility of multiple Zener transitions, since, as a control parameter (a time-dependent charge, for example) is varied, the surfaces repeatedly, and periodically, can approach each other and provide the opportunity for transitions (cf. Fig. 1). In order to discuss multiple Zener transitions, it is important to investigate a more general version of the Landau-Zener problem. They considered a special initial condition, namely, far from the crossing region the system is in one diabatic state with unit probability. Clearly, for multiple transitions a more general initial condition corresponding to a nondiagonal density operator must be considered. The choice of the initial condition profoundly affects the outcome of the transition.
Due to this dependence on initial conditions, the state of the system after consecutive transitions cannot simply be predicted in terms of the properties of a single transition. Even if a given transition has reached its asymptotic region, in the sense that the probability of state occupation has become constant, the off-diagonal elements of the density matrix always oscillate in time, and therefore the result for a new transition will depend on the separation of the transitions. Thus, successive transitions are,

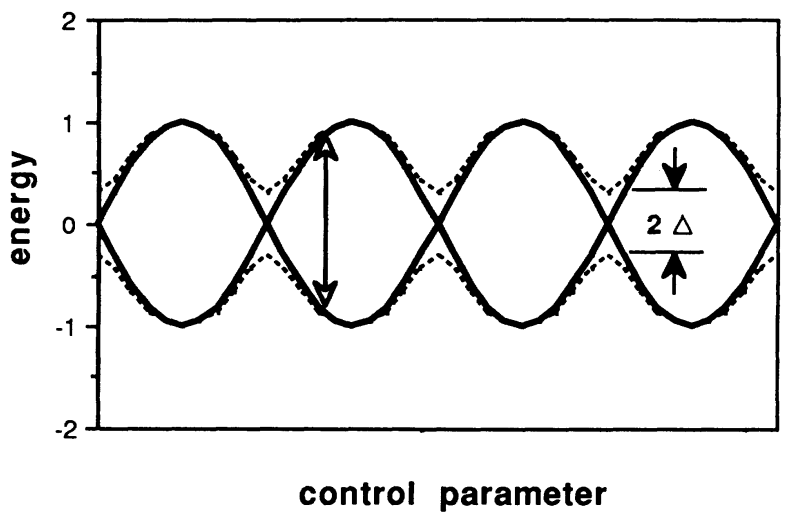

FIG. 1. Energy levels of a two-level system whose Hamiltonian depends on an external control parameter. The solid lines are the diabatic surfaces [the eigenvalues of Eq. (2.1) without the coupling term, $\Delta]$ and the dashed lines are the adiabatic surfaces [the eigenvalues of Eq. (2.1)]. The vertical arrow denotes the system's energy change due to an inelastic transition (cf. Sec. V). 
in general, not independent of each other. We address the multiple-transition problem by numerically solving the equations of motion for the elements of the density matrix of the externally driven system, based on a simple model Hamiltonian. The numerical solution shows that the evolution of the probability is different from that which would be predicted based on a sequence of independent successive Landau-Zener individual transitions. In particular, the latter evolution leads to a probability of one-half for a sufficiently large number of transitions (with the exception of an adiabatic process where the probability oscillates between one and zero) while for the former the probability does not settle down at long times but, rather, keeps oscillating in an involved manner. Clearly, an approach based on independent transitions cannot be correct, due to quantum interference effects.

The behavior of a system undergoing multiple Zener transitions is important to the modeling of electronic devices such as externally-driven-current-biased small tunnel junctions. Here the external source is a current and the response is the voltage output of the junction. The average, dc value $\langle V\rangle$ of the voltage $V$ is an important quantity. As Landauer ${ }^{17}$ pointed out, there is no mechanism for dissipation in a two-level system, even with multiple Zener transitions among the surfaces, therefore $\langle V\rangle=0$ should be obtained. We find this to be the case.

To introduce dissipation into the description, we follow the methodology of other investigators ${ }^{18}$ and incorporate a single-relaxation-time approximation into our equation-of-motion approach. This provides a simple, phenomenological description of dissipation, often used in two-level-system descriptions of magnetic resonance ${ }^{19}$ and optical dephasing. ${ }^{20}$ Here, the nondissipative Hamiltonian is time dependent, so the dissipative transitions provide a relaxation mechanism to a time-dependent state. The numerical solution of the resulting equations of motion is readily obtained and provides the time evolution of the probability and voltage. Now that there is a mechanism for dissipation, we find that the average voltage $\langle V\rangle$ is no longer zero. Though, as relaxation becomes rapid, relative to the time scale of motion along the external coordinate, $\langle V\rangle$ approaches zero again.

The plan of the rest of this paper is as follows. In Sec. II we introduce the model Hamiltonian and obtain the coupled equations of motion for the elements of the density matrix. Section III considers the single-crossing, linear model (the Landau-Zener model) from the equation-of-motion perspective. This model was recently analyzed by Mullen et al. ${ }^{21}$ by a combination of analytic and numerical methods. They evaluated the time course of probability (versus asymptotic probability) for the linear Landau-Zener model with the conventional unit probability initial condition. The results we obtain are in accord with theirs, and serve as a check of our methodology. We then evaluate the results of a transition for more general initial conditions. In Sec. IV we formulate and analyze the multiple-Zener-crossing model and show how the independent single-Zener-transition predictions fail. The effects of dissipation on multiple Zener transitions are investigated in Sec. $\mathrm{V}$.

\section{FORMALISM}

We will deal with the model Hamiltonian

$$
H=\phi(t) s_{z}+\Delta s_{x}
$$

describing the dynamics of a two-state system driven by a time-dependent term $\phi(t)$, characterizing the action of some external agent. The $s_{\alpha}(\alpha=x, y, z)$ are the usual Pauli matrices, which in the basis of eigenstates of $s_{z}$ $\left(s_{z}|1\rangle=|1\rangle ; s_{z}|2\rangle=-|2\rangle\right)$ have the matrix representations

$$
\begin{aligned}
& s_{z}=|1\rangle\langle 1|-| 2\rangle\langle 2|=\left[\begin{array}{cc}
1 & 0 \\
0 & -1
\end{array}\right], \\
& s_{x}=|1\rangle\langle 2|+| 2\rangle\langle 1|=\left[\begin{array}{ll}
0 & 1 \\
1 & 0
\end{array}\right], \\
& s_{y}=i(|2\rangle\langle 1|-| 1\rangle\langle 2|)=\left[\begin{array}{cc}
0 & -i \\
i & 0
\end{array}\right] .
\end{aligned}
$$

If $\Delta=0$, and the system is initially in one eigenstatesay, in state $|1\rangle$-it will remain in this state forever. But, if $\Delta$ is not negligible, then sooner or later there will be a probability for finding the system in state $|2\rangle$, as states $|1\rangle$ and $|2\rangle$ are no longer eigenstates of the whole Hamiltonian. A further complication arises because the Hamiltonian itself is time dependent. If $H$ varies very slowly in time, one can resort to the adiabatic approximation scheme $e^{22}$ to deal with the problem of calculating the probability of transition between states.

Here, we will follow another procedure which we believe is more efficient from a computational point of view. Let $|\Psi(t)\rangle$ describe the state of the system at time $t$. We are interested in the probability $P_{1}(t)$ that the system will be found in state $|1\rangle$ at time $t$, which in terms of the expectation value of $s_{z}$ is given by

$$
P_{1}(t)=|\langle\Psi(t) \mid 1\rangle|^{2}=\frac{1}{2}\left[1+\left\langle s_{z}(t)\right\rangle\right] \text {. }
$$

In order to calculate the quantity $\left\langle s_{z}(t)\right\rangle$, we use the Schrödinger equation. Because $H$ is time dependent, $\left[H\left(t_{1}\right), H\left(t_{2}\right)\right] \neq 0$ for $t_{1} \neq t_{2}$. Therefore, we write

$$
|\Psi(t)\rangle=U(t)|\Psi(0)\rangle
$$

where $U(t)$ denotes the following unitary operator: ${ }^{23}$

$$
U(t)=T_{+} \exp \left[-(i / \hbar) \int d t^{\prime} H\left(t^{\prime}\right)\right]
$$

and $T_{+}$is a time-ordering operator with the largest time on the left. Then

$$
\left\langle s_{z}(t)\right\rangle=\left\langle\Psi(t)\left|s_{z}\right| \Psi(t)\right\rangle=\left\langle\Psi\left(t_{0}\right)\left|U^{\dagger} s_{z} U\right| \Psi\left(t_{0}\right)\right\rangle
$$

obeys the equation motion

$$
\begin{aligned}
i \hbar \frac{\partial}{\partial t}\left\langle s_{z}(t)\right\rangle & =\left\langle\Psi\left(t_{0}\right)\left|U^{\dagger}\left[s_{z}, H_{s}(t)\right] U\right| \Psi\left(t_{0}\right)\right\rangle \\
& =2 i \Delta\left\langle s_{y}(t)\right\rangle .
\end{aligned}
$$

In obtaining Eq. (2.6), we use

$$
i \hbar \frac{\partial}{\partial t} U=H_{s} U, \quad-i \hbar \frac{\partial}{\partial t} U^{\dagger}=U^{\dagger} H_{s}
$$


and the commutation relations of the Pauli matrices $\left[s_{\alpha}, s_{\beta}\right]=2 i s_{\gamma}, \alpha \neq \beta \neq \gamma \neq \alpha$. Proceeding along similar lines, one can get an evolution equation for $\left\langle s_{x}(t)\right\rangle,\left\langle s_{y}(t)\right\rangle$, so we have the system of equations

$$
\begin{aligned}
& \left\langle\dot{s}_{x}(t)\right\rangle=-\frac{2 \phi(t)}{\hbar}\left\langle s_{y}(t)\right\rangle, \\
& \left\langle\dot{s}_{y}(t)\right\rangle=\frac{2 \phi(t)}{\hbar}\left\langle s_{x}(t)\right\rangle-\frac{2 \Delta}{\hbar}\left\langle s_{z}(t)\right\rangle, \\
& \left\langle\dot{s}_{z}(t)\right\rangle=\frac{2 \Delta}{\hbar}\left\langle s_{y}(t)\right\rangle,
\end{aligned}
$$

where the overdot denotes time derivative. The solution of this system of equations with given initial condition allows us to find the quantity $P_{1}(t)$.

An alternative formulation, which will be useful later, is based on the use of the system density operator $\rho(t)$ satisfying

$$
i \hbar \frac{\partial}{\partial t} \rho(t)=[H(t), \rho(t)]
$$

If we expand

$$
\rho(t)=\frac{1}{2}\left[1+x(t) s_{x}+y(t) s_{y}+z(t) s_{z}\right]
$$

and substitute into Eq. (2.9), we obtain Eqs. (2.8) with $\left\langle s_{x}(t)\right\rangle,\left\langle s_{y}(t)\right\rangle$, and $\left\langle s_{z}(t)\right\rangle$ replaced by $x(t), y(t)$, and $z(t)$, respectively, and the probability $P_{1}(t)$ given as

$P_{1}(t)=\operatorname{tr} \rho(t)|1\rangle\langle 1|=\langle 1|\rho(t)| 1\rangle=\frac{1}{2}[1+z(t)]$.

The quantities $x(t), y(t)$, and $z(t)$ are related to the wave function $|\Psi\rangle$ of the system as follows. In the diabatic basis, we write $|\Psi(t)\rangle=a(t)|1\rangle+b(t)|2\rangle$. Recalling that the density operator $\rho(t)=|\Psi(t)\rangle\langle\Psi(t)|$ and writing the spin operators in terms of $|1\rangle\langle 1|$ and $|2\rangle\langle 2|$, we can identify the complex numbers $a(t)$ and $b(t)$ as $a(t)=r_{a}(t) \exp \left[i \phi_{a}(t)\right]$ and $b(t)=r_{b}(t) \exp \left[i \phi_{b}(t)\right]$ with $r_{a}^{2}(t)=P_{1}(t), \quad r_{b}^{2}(t)=1-P_{1}(t), \quad$ and $\tan \left(\phi_{a}-\phi_{b}\right)$ $=-y(t) / x(t)$. We now consider several different situations.

\section{SINGLE-CROSSING LINEAR MODEL (LANDAU-ZENER)}

Let us consider a situation where $\phi(t)=\alpha t$ with $\alpha$ a constant. The Hamiltonian

$$
H=\alpha t s_{z}+\Delta s_{x}
$$

describes the crossing at $t=0$ of two linear diabatic surfaces $\alpha t$ and $-\alpha t$. The problem analyzed by Landau and Zener assumes that the system was in state $|1\rangle$ for $t \rightarrow-\infty$. Introducing an adiabaticity parameter, $\gamma=\hbar \alpha / \Delta^{2}$, the probability $P_{1}(t)$ has the asymptotic value

$$
P_{1}(t) \sim e^{-\pi / \gamma} \text { as } t \rightarrow \infty .
$$

For $\gamma \ll<1$, the crossing is said to be adiabatic and the asymptotic probability $P_{1}(\infty)$ goes to zero. This simply expresses the fact that the coupling between the diabatic surfaces is so strong that the system will certainly jump to state $|2\rangle$ at the crossing region. In other words, the system will almost certainly follow the lower adiabatic surface (cf. Fig. 1).

The duration of the adiabatic transition can be estimated by noticing that the transition is possible for those times for which the tunneling energy $\Delta$ is able to lift the degeneracy at the crossing, i.e., for $\alpha \tau_{Z a} \sim \Delta$. This defines the time scale for the duration of the adiabatic crossing.

For $\gamma \gg 1$, the transition is called sudden or nonadiabatic. The probability $P_{1}(\infty) \simeq 1$ and so the probability of transferring to state $|2\rangle$ is very small. In terms of the adiabatic surfaces, the system will jump from one surface to the other with a large probability. To estimate the tunneling time, one can use the Heisenberg relation and the fact that $\Delta$ is always very small to write $\Delta E \tau_{Z} \sim \hbar$ and $\Delta E \sim 2 \alpha \tau_{Z}$. Thus, $\tau_{Z d} \sim(\hbar / \alpha)^{1 / 2}$, which is the time scale for a nonadiabatic transition, as noted by Mullen et al. ${ }^{21}$ More generally, a characteristic time $\tau_{Z}$ of passage of the crossing region can be obtained from the separation between the turning points of the motion. ${ }^{24}$ For the linear crossing model, we define

$$
\tau_{Z}=(\Delta / a)\left(1+\gamma^{2}\right)^{1 / 4},
$$

where, for $\gamma \ll<1, \tau_{Z} \rightarrow \tau_{Z a}$ and for $\gamma \gg 1, \tau_{Z} \rightarrow \tau_{Z d}$.

When considering the duration of a transition, attention also has to be paid to the off-diagonal elements of the density operator $x$ and $y$. It is convenient to scale the time variable to the dimensionless variable $u=(\alpha / \hbar)^{1 / 2} t$, in terms of which Eqs. (2.8) read

$$
\begin{aligned}
& \frac{d x(u)}{d u}=-2 u y, \\
& \frac{d y(u)}{d u}=-2 u x-\frac{2}{\sqrt{\gamma}} z, \\
& \frac{d z(u)}{d u}=\frac{2}{\sqrt{\gamma}} y .
\end{aligned}
$$

For times $u>2 / \sqrt{\gamma}$, i.e., for times such that the system is away from the transition region and the probability has almost reached its asymptotic constant value, the offdiagonal elements can be approximated by the solution of $d x / d u=-2 u y ; d y / d u=2 u x$, which is

$$
\begin{aligned}
& x(u)=x_{i n} \cos u^{2}-y_{i n} \sin u^{2}, \\
& y(u)=x_{i n} \sin u^{2}-y_{i n} \cos u^{2} .
\end{aligned}
$$

This indicates that $x$ and $y$ do not decay but rather that they show an oscillatory behavior. The amplitude of the oscillations is dictated by the fact that

$$
x^{2}(u)+y^{2}(u)+z^{2}(u)=1
$$

is an exact first integral of Eq. (2.8). Thus, for an adiabatic transition, say, $\gamma=0.2, z(u)$ for large $u$ is almost -1 if the system started at $u \rightarrow-\infty$ with $x=y=0$ and $z=1$ corresponding to the system initially in a diabatic state. Then $x^{2}+y^{2} \approx 0$, and therefore $x$ and $y$ oscillate with very small amplitudes. On the other hand, for a nonadiabatic transition $(\gamma>1) z(u)$ for large $u$ differs from 1 , and $x$ and $y$ will show large amplitude oscillations. 

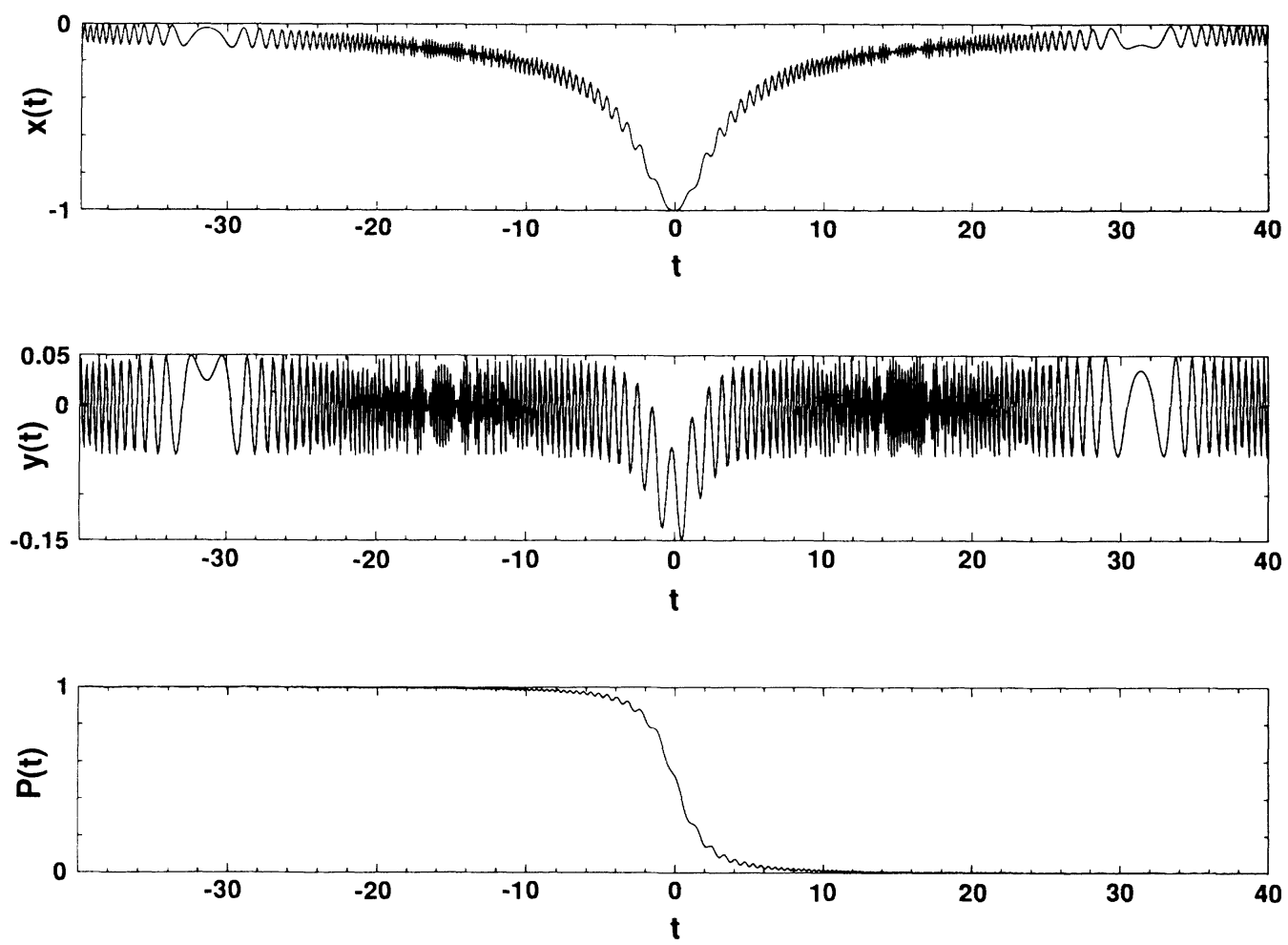

FIG. 2. $x(t), y(t)$, and $P_{1}(t)$ for $\gamma=0.2$ with initial conditions $x_{i n}=y_{i n}=0.0$ and $z_{i n}=1.0$.

Since successive transitions will have arbitrary initial values of $x, y$, and $z$ [obeying Eq. (3.6)], we must analyze the dynamics of a single transition for these nonstandard initial conditions. In order to do this, the system of equa- tions [Eq. (2.8)], with $\phi(t)=\alpha t$, was integrated numerically by means of a fourth-order Runge-Kutta procedure. ${ }^{25}$ We have chosen an integration time step such that for all times, condition (3.6) is satisfied. The results for $P(t)$ for
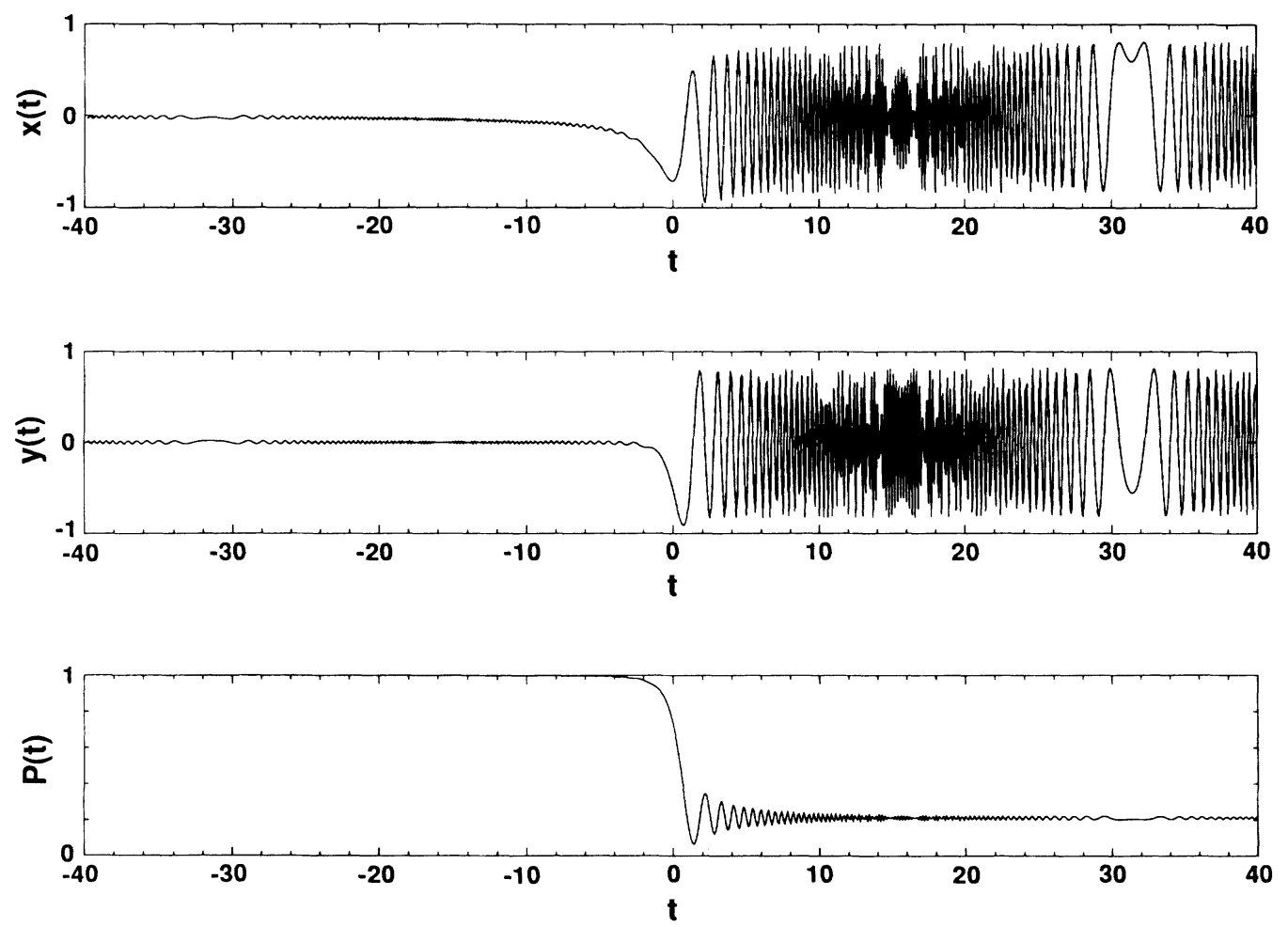

FIG. 3. $x(t), y(t)$, and $P_{1}(t)$ for $\gamma=2.0$ with initial conditions $x_{i n}=y_{i n}=0.0$ and $z_{i n}=1.0$. 
TABLE I. The asymptotic transition probability $P_{1}(\infty)$ for different initial conditions. Here $z_{\text {in }}=0.0$ (equal initial populations in both states) always, and the initial phases are determined by the below $x_{i n}$ and $y_{\text {in }}$ combinations.

\begin{tabular}{rlll}
\hline$\gamma$ & $x_{\text {in }}$ & $y_{\text {in }}$ & $P_{1}(\infty)$ \\
\hline 0.2 & 1.0 & 0.0 & 0.5 \\
0.2 & 0.0 & 1.0 & 0.5 \\
2.0 & 1.0 & 0.0 & 0.12 \\
2.0 & 0.0 & 1.0 & 0.61 \\
20.0 & 1.0 & 0.0 & 0.75 \\
20.0 & 0.0 & 1.0 & 0.75 \\
20.0 & 0.707 & 0.707 & 0.85 \\
20.0 & 0.707 & -0.707 & 0.49 \\
20.0 & -1.0 & 0.0 & 0.26 \\
\hline \hline
\end{tabular}

different values of $\gamma$ with the initial condition $x=y=0$ and $z=1$ agree with the Landau-Zener asymptotic probability formula of Eq. (3.2) and with the results of Mullen et al. ${ }^{21}$ In Figs. 2 and 3, we plot the results for $x, y$ and $P$ as given by the numerical solution for two different values of $\gamma$. As discussed above, away from the adiabatic limit, the oscillations of $x$ and $y$ are substantial after the transition has occurred. Note that the modulation in amplitude of the $x$ and $y$ oscillations shown in these figures is not real but an artifact due to the fact that we are plotting our data every 100 time steps (time step $=0.001$ ).

A selection of asymptotic probabilities $P(\infty)$ for equal initial probability in both levels $\left(z_{i n}=0.0\right)$ and several initial condition combinations of $x_{i n}$ and $y_{\text {in }}$ are presented in Table I. For the adiabatic case $(\gamma=0.2)$, where the oscillations in $x$ and $y$ are small, a formula for the transition probability based on probabilistic arguments is readily obtained. If $r$ denotes the transition probability assuming the Zener formula of Eq. (3.2), then weighing this probability with the initial-state occupations leads to

$$
P(\infty)=r P_{i n}+(1-r)\left(1-P_{\text {in }}\right),
$$

where $P_{\text {in }}=\left(z_{\text {in }}+1\right) / 2$. For example, with $P_{\text {in }}=0.5$ $\left(z_{\text {in }}=0.0\right)$ as chosen above, $P(\infty)=0.5$, in agreement with the results of the numerical solution for the adiabatic case. Away from this adiabatic limit, probabilistic prescriptions fail as the quantum interference between the wave functions on the two surfaces becomes critical in determining the asymptotic probabilities.

The above results show that the initial values of the off-diagonal elements of the density operator have to be taken into account for the prediction of the asymptotic probability as well as for the initial conditions required for any subsequent transition. These features are important when considering consecutive transitions, as we will analyze in the next section.

\section{NONLINEAR MODEL: MULTIPLE CROSSINGS}

We now consider the case $\phi(t)=A \sin (\omega t)$, where $\omega$ represents the externally applied "force" (bias). The energy levels of the Hamiltonian (the adiabatic levels) are then

$$
\lambda_{+}=\sqrt{\Delta^{2}+A^{2} \sin ^{2}(\omega t)}, \quad \lambda_{-}=-\lambda_{+} .
$$

Away from the crossing points, the $\lambda_{ \pm}$coincide with $\phi$, while, near the crossings, the adiabatic energy levels split apart by $2 \Delta$ (cf. the dotted line on Fig. 1).

The diabatic energy levels $\pm \phi(t)$, which are the solution of $H_{0}=\phi(t) s_{z}$, are also shown in Fig. 1. If motion is along one of the adiabatic levels - say, the lower onethe system goes along, alternatively, both the diabatic energy levels. This indicates that the probability of transition from one diabatic level to another at each crossing is very large. Thus, the transition at each crossing is adiabatic.

The structure of a two-level system with energy levels showing a periodic gap structure, as depicted in Fig. 1, is reminiscent of the model used by Landauer and Büttiker ${ }^{26}$ in the context of the analysis of small metallic loops driven by time-dependent magnetic fluxes, or by Büttiker $^{18}$ and other works $^{9-12,21}$ in the context of current-driven small tunnel junctions.

The main feature of the model lies precisely in the periodic structure of the energy levels and, therefore, on the fact that, as time increases, the system goes through many consecutive Zener-transition opportunities. By numerical solution of the equations of motion, we can then address the question of how the probabilities for consecutive transitions are influenced by the time evolution of the off-diagonal elements of the density matrix. Notice that, if the Hamiltonian is linearized around a crossing point of the diabatic surfaces, one is back to the linear model for that particular crossing, with $\alpha=A \omega$. Thus, it is still useful to define an adiabaticity parameter $\gamma=A \omega \hbar / \Delta^{2}$, as in Sec. III, and a corresponding Zener time $\tau_{Z}$, as in Eq. (3.3).

The results are shown in Figs. 4-6 for different values of the adiabaticity parameter $\gamma$. For a small value of $\gamma$ $(\gamma=0.2), P_{1}$ oscillates between 0 and 1 so that the system always remains on the lower adiabatic surface (Fig. 4). This result can be rationalized by an extension of the probabilistic argument presented in Sec. III, as we now show.

Assuming that interference effects can be neglected, so that the dynamics for successive transitions can be described solely in terms of probabilities, we can write the following equation for $P_{1}\left(t_{n}\right)$ :

$$
P_{1}\left(t_{n}\right)=r P_{1}\left(t_{n-1}\right)+(1-r)\left[1-P_{1}\left(t_{n-1}\right)\right],
$$

where $r$ again is the Landau-Zener probability of ending up in the same state as the one we started from at each crossing, and $P_{1}\left(t_{n-1}\right)$ is the probability of being in state 11) after $(n-1)$ crossing regions have been traversed. Iteration of Eq. (4.2) leads to

$$
P_{1}\left(t_{n}\right)=(2 r-1)^{n} P_{1}\left(t_{0}\right)+\left(\frac{1}{2}-\frac{(2 r-1)^{n}}{2}\right) \text {, }
$$

where $P_{1}\left(t_{0}\right)$ is the probability to be in state $|1\rangle$ at some initial time $t_{0}$. If we take $P_{1}\left(t_{0}\right)=1$, then we have

$$
P_{1}\left(t_{n}\right)=\frac{(2 r-1)^{n}}{2}+\frac{1}{2} \text {. }
$$



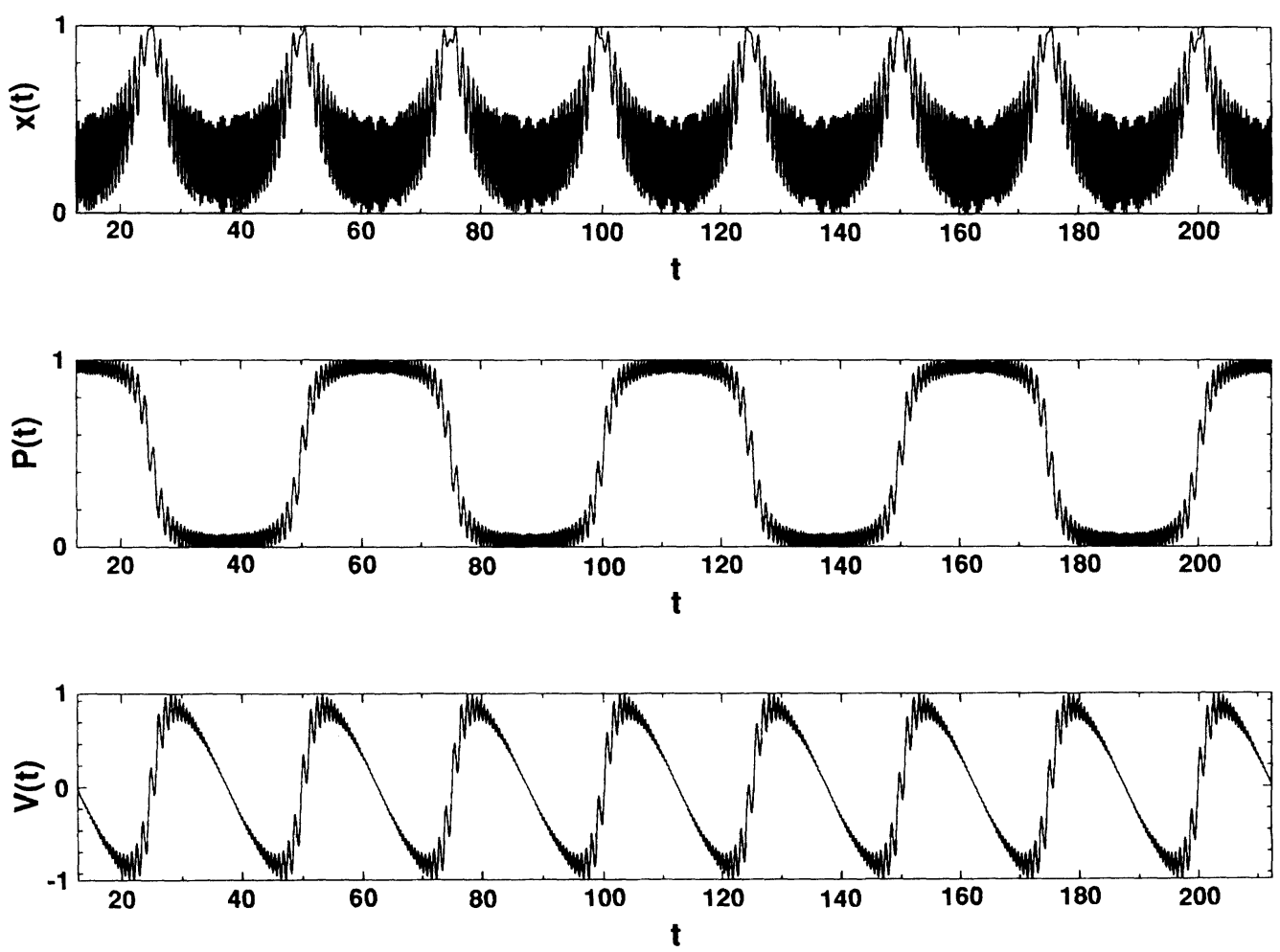

FIG. 4. $x(t), P(t)$, and the voltage $V(t)$ for multiple transitions with the conditions of Fig. 2 .

If $r=0$, then $P_{1}\left(t_{n}\right)=0$ (1) for $n$ odd (for $n$ even). Thus, for adiabatic transitions, Eq. (4.4) will approximate the solution of the equations of motion for some time interval.
For values of $\gamma$ away from the adiabatic regime, the probabilistic formula of Eq. (4.4) is not correct. The numerical results displayed in Figs. 5 and 6 show that, in contrast to the prediction of Eq. (4.4) where the probabil-
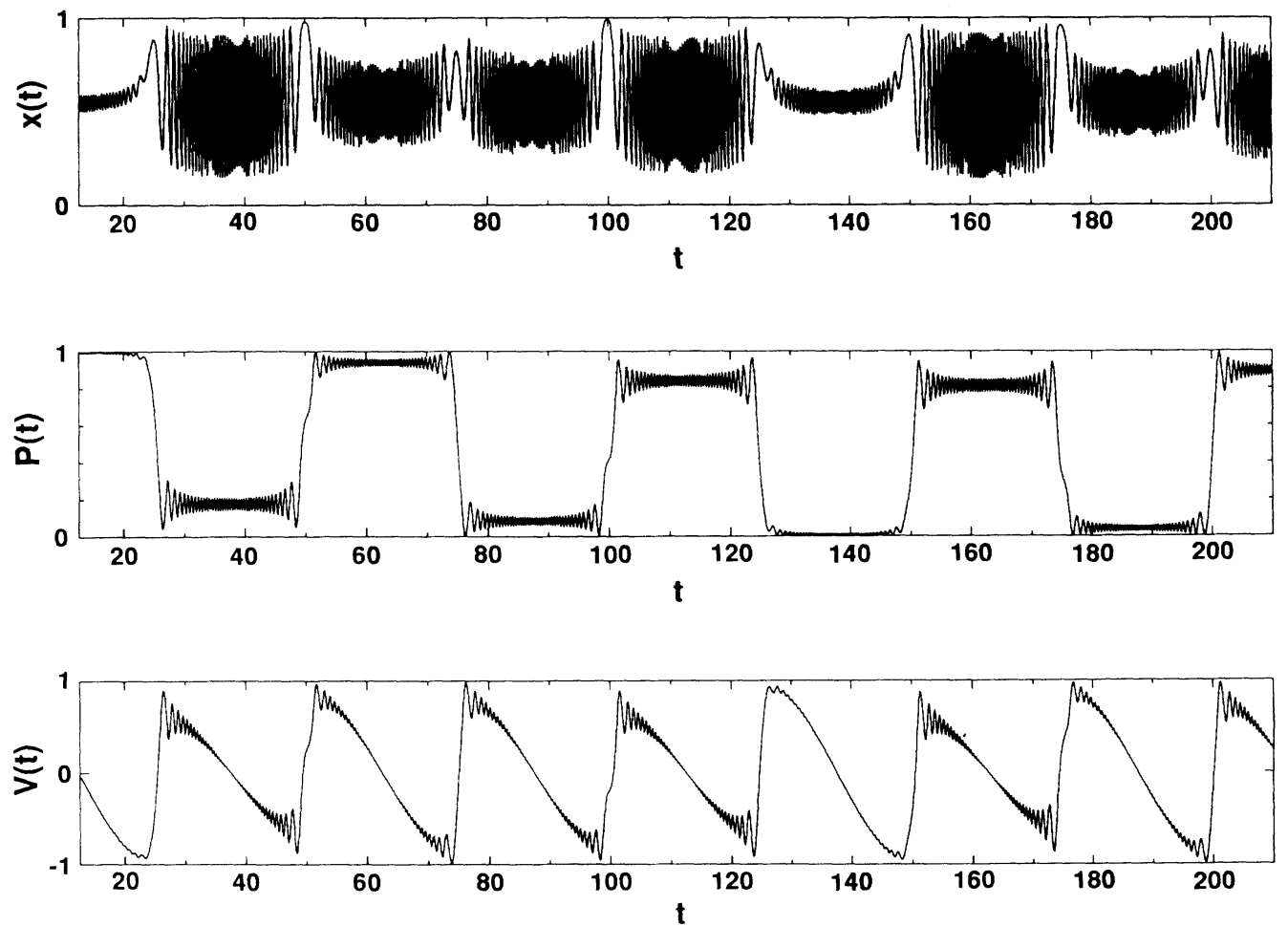

FIG. 5. $x(t), P(t)$, and the voltage $V(t)$ for multiple transitions with the conditions of Fig. 3 . 


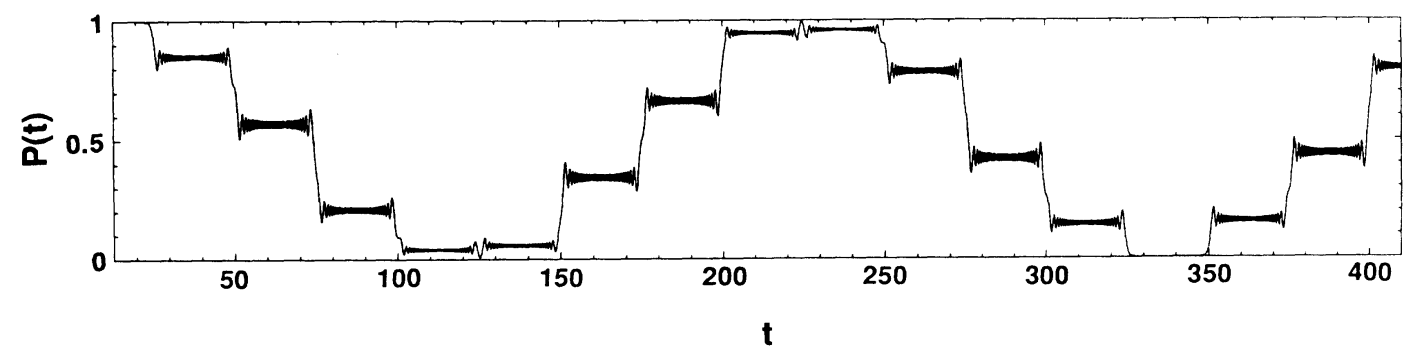

FIG. 6. $P(t)$ for multiple transitions for $\gamma=20.0$ and $x_{i n}=y_{i n}=0.0$ and $z_{i n}=1.0$.

ity eventually reaches a limit of 0.5 , the probability oscillates indefinitely. The actual pattern of probability for the multiple transitions will depend on the choice of the initial conditions $x_{i n}, y_{i n}$, and $z_{i n}$, and the separation in time of the transition regions. Even if the transitions are separated sufficiently in time such that the probabilities have reached their asymptotic values after a given transition, the oscillations in $x(t)$ and $y(t)$ will provide an "initial condition" for the next transition that depends on the time separation of the transitions. Thus, if an analytic expression for the multiple-transition case is desired, not only would the solution of the extended Landau-Zener problem for all the elements of the density matrix from an arbitrary initial condition be required, but also a method of matching the outgoing and incoming density matrices, based on an expression for the evolution of $x(t)$ and $y(t)$ away from the transition regions similar to those given in Eq. (3.5), would have to be developed.

\section{THE EFFECT OF INELASTIC TRANSITIONS}

That Zener transitions alone are not capable of producing dissipation has been already pointed out by Landauer and Büttiker. ${ }^{17,18,26}$ This feature is clear from the results of the preceding section. Of course, if Eq. (4.4) was used, then a finite time-independent $P_{1}(t)$ would be predicted at long times (for $r \neq 0$ or 1 ), because one would have built in an extra assumption, namely, that only probabilities contribute to the dynamics.

In this section we consider the consequences of adding inelastic-transition events to the multiple-Zener-crossing model. Let us assume that the two levels are interacting with some other degrees of freedom such that a vertical transition represented by the vertical arrow on Fig. 1 is possible. Since the system's energy changes in the course of the transition, it is an inelastic event from the system's point of view.

Inelastic transitions have been considered before, when discussing the resistance of small metallic loops ${ }^{10}$ as well as in the context of a small-capacitance Josephson junction. ${ }^{18}$ They provide a mechanism for dissipation, which is required in order to observe a nonzero time-average response of the system to a constant, externally applied force, e.g., a dc component $(\langle V\rangle \neq 0)$ to the voltage response of a current-driven, small Josephson junction.

For the sake of definiteness, let us use the language of a current-driven device. Then, $\omega=I / e$ is the current in units of charge and the corresponding charge is $q=I t$. The quantity $\phi(t)$ is given as

$$
\phi(t)=A \sin (\omega t)=E_{c} \sin q / e,
$$

where $E_{c}=e^{2} / 2 C$ is the charging energy of a junction of capacitance $C$. Define the voltage operator as $\hat{\mathbf{v}}=\partial H / \partial q$. The expectation value $V(t)$ of $\checkmark$ is given by

$$
V(t)=\operatorname{tr}[\hat{\rho}(t) \hat{\mathbf{v}}] .
$$

Taking into account Eq. (2.1) and the definition $\left\langle s_{z}(t)\right\rangle=z(t)$ [cf. after Eq. (2.9)], we find that

$$
V(t)=\left(E_{c} / e\right) \cos (I t / e) z(t) .
$$

We now incorporate, in an approximate way, both the effects of inelastic transitions and the dynamical effects arising from the system's own Hamiltonian. Let us define the quantity

$$
\rho_{0}(t)=\frac{1}{Z_{0}(t)} e^{-\beta H(t)},
$$

where $\beta=\left(k_{B} T\right)^{-1}$ and $T$ is the temperature. The quantity $\rho_{0}(t)$ represents the (time-dependent) canonical equilibrium distribution that would eventually prevail as a consequence of the inelastic transitions. If Zener transitions are neglected, a phenomenological way to describe the relaxation of the density operator toward this state is by means of the kinetic equation

$$
\frac{\partial \hat{\rho}}{\partial t}=-\frac{1}{\tau}\left[\hat{\rho}(t)-\hat{\rho}_{0}\right],
$$

where $\tau$ is a relaxation time. In general, $\tau$ depends on temperature and on the other parameters. We will consider it as a constant here. To incorporate the effects of the Zener transitions, we propose the kinetic equation

$$
\frac{\partial \hat{\rho}}{\partial t}=\frac{1}{i \hbar}[H(t), \hat{\rho}]-\frac{1}{\tau}\left[\hat{\rho}(t)-\hat{\rho}_{0}\right] .
$$

This neglects any dynamical interference between the two types of effects: Zener and inelastic transitions.

For the Hamiltonian of Eq. (4.5), it is straightforward to explicitly calculate $\rho_{0}(t)$,

$$
\begin{array}{r}
\hat{\rho}_{0}(t)=\frac{1}{2}\left[\begin{array}{l}
1-\frac{\Delta \tanh \left(\beta \sqrt{\Delta^{2}+\phi^{2}}\right)}{\sqrt{\Delta^{2}+\phi^{2}}} s_{x} \\
-\frac{\phi \tanh \left(\beta \sqrt{\Delta^{2}+\phi^{2}}\right)}{\sqrt{\Delta^{2}+\phi^{2}}} s_{z}
\end{array}\right],
\end{array}
$$

where $\phi(t)$ is given by Eq. (5.1). Inserting Eq. (5.7) into 

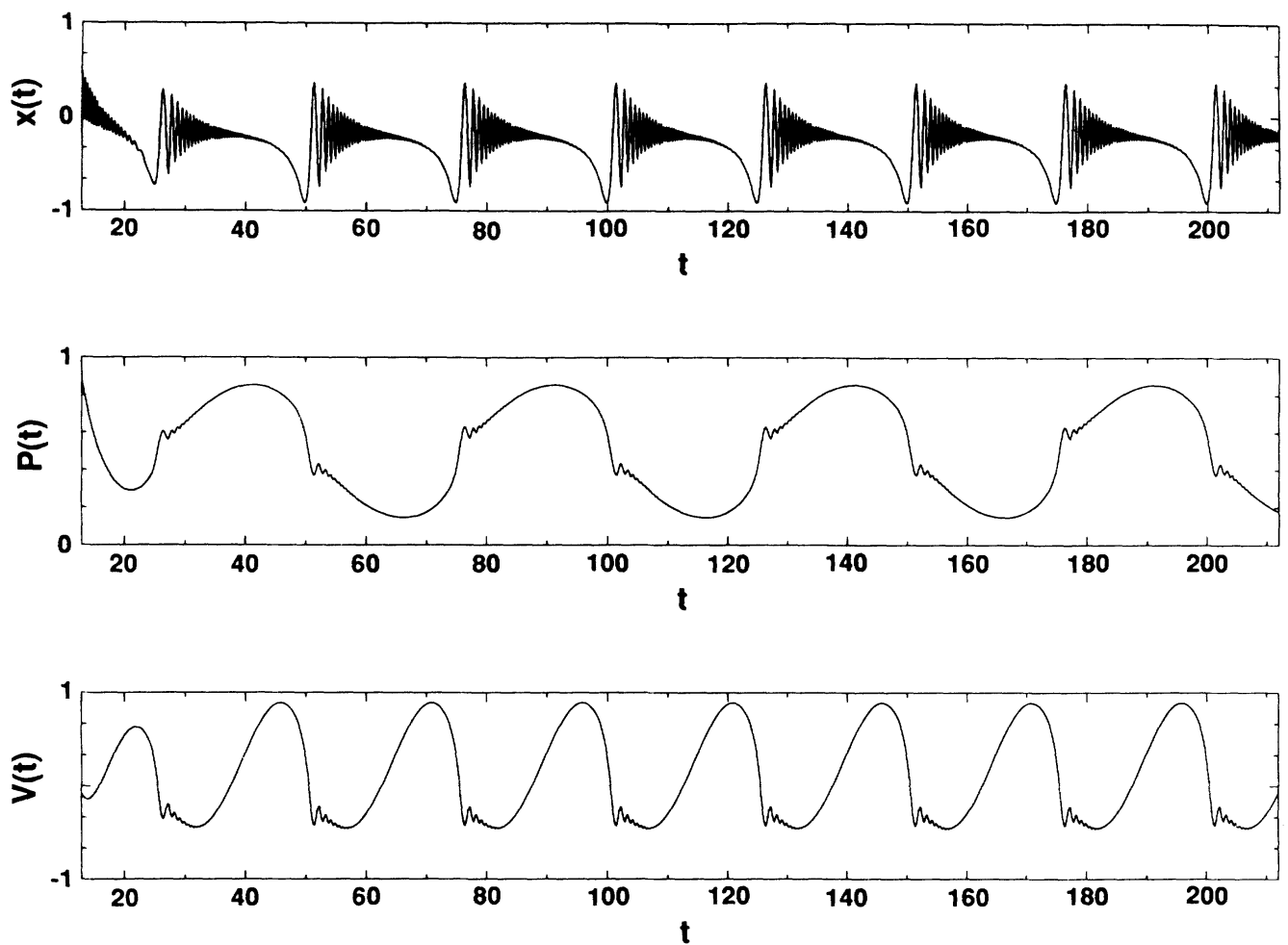

FIG. 7. $x(t), P(t)$, and the voltage $V(t)$ for $\gamma=20.0$ and an inelastic transition time $\tau$ such that $2 I \tau / e=1.0$. The dc voltage $\langle V\rangle$ is not zero.

Eq. (5.6), we obtain the equations of motion

$$
\begin{aligned}
\dot{x}(t)= & -\frac{2 \phi(t)}{\hbar} y(t) \\
& -\frac{1}{\tau}\left[x(t)+\frac{\Delta}{\sqrt{\Delta^{2}+\phi^{2}}} \tanh \left(\beta \sqrt{\Delta^{2}+\phi^{2}}\right)\right] \\
\dot{y}(t)= & -\frac{2 \phi(t)}{\hbar} x(t)-\frac{2 \Delta}{\hbar} z(t)-\frac{1}{\tau} y(t) \\
\dot{z}(t)= & -\frac{2 \Delta}{\hbar} y(t) \\
& -\frac{1}{\tau}\left[z(t)+\frac{\phi(t) \tanh \left(\beta \sqrt{\Delta^{2}+\phi^{2}}\right)}{\sqrt{\Delta^{2}+\phi^{2}}}\right)
\end{aligned}
$$

This system of equations is readily solved numerically. If we assume that the system was initially in state $|1\rangle$, then the appropriate initial conditions are $x\left(t_{\text {in }}\right)=y\left(t_{\text {in }}\right)=0$, $z\left(t_{\text {in }}\right)=1$.

For large $\tau$, the contribution from inelastic transitions can be neglected. Equations (5.8) then reduce to Eqs. (2.8) describing the Zener transitions. If $\gamma$ is small, then the system follows the lower surface and the voltage $\sim \partial \lambda_{-} / \partial q$. Thus, both $z(t)$ and $V(t)$ are periodic with frequency $\omega=I / e$. Figure 4 illustrates this behavior for $\gamma=0.2$. Figure 5 displays the voltage oscillations for $\gamma=2.0$. They mirror the oscillations of $P(t)$. The pattern of the voltage oscillations becomes more irregular in appearance as the adiabaticity parameter $\gamma$ is increased. In all these cases, the average dc voltage is zero since, with $\tau \rightarrow \infty$, there is no possibility for dissipation.
For $\tau \rightarrow 0$, the effect of Zener transitions is negligible. The system rapidly relaxes to $\rho_{0}(t)$, and, therefore, we have from Eqs. (5.1), (5.3), and (5.8) that

$$
\begin{aligned}
V(t) & =-\frac{1}{I} \dot{\phi}(t) \phi(t) \frac{\tanh \left(\beta \sqrt{\Delta^{2}+\phi^{2}}\right)}{\Delta^{2}+\phi^{2}} \\
& =\frac{-1}{I} \frac{\partial}{\partial t} \ln \cosh \left(\beta \sqrt{\Delta^{2}+\phi^{2}}\right) .
\end{aligned}
$$

The quantity $\left(\Delta^{2}+\phi^{2}\right)^{1 / 2}$ is periodic. Thus, the time average of $V(t),\langle V(t)\rangle$, also yields a zero dc component.

When $\tau$ is finite, the average voltage no longer is zero. In Fig. 7 we display the time-dependent voltage for

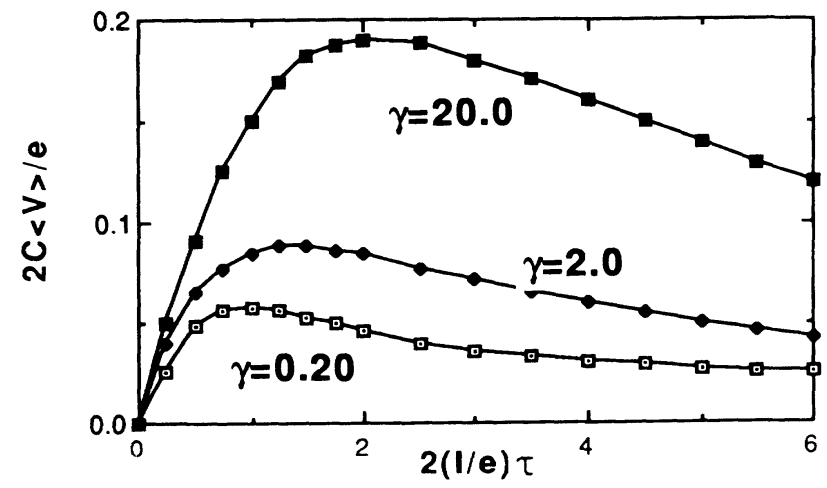

FIG. 8. The dc voltage $\langle V\rangle$ plotted vs the product of the applied current $I$ and the inelastic relaxation time $\tau$, for a series of $\gamma$ values. 
$\gamma=2.0$ and $2 I \tau / e=1.0$. The voltage oscillations are similar to those for $\gamma=0.2$ and $\tau \rightarrow \infty$, but here the dc voltage is clearly not zero. The probability never approaches a constant value, as it does for completed transitions in the absence of inelastic processes, since it is always being driven toward its time-dependent equilibrium form, as obtained from Eq. (5.4). Furthermore, $x(t)$ exhibits oscillations that are damped to values close to zero. This behavior shows the interplay between the oscillating and damping terms in Eqs. (5.8) for $x(t)$ and $y(t)$, for times away from the coupling regions.

In Fig. 8 we plot $I \tau$ versus the average voltage $\langle V\rangle$ for several fixed values of $\gamma$. Since $\gamma \sim I$, keeping $I$ fixed also fixes $\gamma$, and Fig. 8 then reveals the effect of different $\tau$ values on the average voltage for a series of constant current values.

\section{CONCLUSION}

In this work we have developed an efficient numerical method to analyze the problem of single and multiple Zener transitions. This method allows us to clarify the role of arbitrary initial condition in the time evolution of a system going through a region coupling two diabatic surfaces. In particular, initial conditions different from those conventionally chosen within the context of the Landau-Zener model have been investigated, as an aid in rationalizing the results for multiple Zener transitions. The results demonstrate that an approach in terms of probabilities alone cannot in general describe the outcome of a Zener transition. This is apparent as all the elements of the density matrix contribute to the system's time evolution. Thus, in describing multiple Zener transitions, the evolution of all the density-matrix elements must be accounted for, especially as $\gamma$ increases toward the nonadiabatic regime.

Note that for a given value of $\gamma$ and separation of the transitions, the system's time evolution, for all times, depends on the initial conditions. Thus, a simple probabilistic expression, as given in Eq. (4.4), is not correct for multiple transitions. If the transitions were separated in the sense that each had reached its asymptotic probabili- ty before the next transition started, then it would be possible to construct an expression for the time evolution of the probability for multiple Zener transitions. This would require an analytic expression for a generalized Landau-Zener formula for a single transition and a knowledge of the separation of the transitions. The generalized Landau-Zener formula, along with the analog of Eqs. (3.5) for the sine model to propagate the system between transitions, would then permit evaluation of the multiple-Zener-transition probabilities. Clearly, this would be a complicated undertaking.

The inclusion of inelastic transitions modifies the Zener transition results in a profound manner. For currentdriven small tunnel junctions, the voltage output still oscillates but now the average, dc voltage is nonzero. Thus, the inclusion of a dissipative relaxation mechanism leads to a nonzero dc voltage.

In work not reported here, ${ }^{27}$ we also considered the case where the frequency $\omega$ is a stochastic variable. For example, in a current-driven junction $\omega=I / e$. In this case, a stochastic component to the current $I$ would arise from an external noise source. We found that both $P_{1}(t)$ and $V(t)$ are quite insensitive to this randomness, which reflects the stability of these driven systems against current fluctuations.

Above, we have stressed the relevance of the methods developed in this work to small normal and Josephson tunnel junctions. In these instances and many others, ${ }^{21}$ the two-level bandlike structure of Fig. 1 is augmented by additional levels on top of those displayed there. We are currently adapting the methodology presented herein to be applicable to this multilevel multiple-Zener-transition problem.

\section{ACKNOWLEDGMENTS}

M.M. and J.M.C. acknowledge the Dirección General de Investigación Científica y Técnica (DGICYT) of Spain (Project No. PS89-0123) for financial support. R.I.C. and M.M. acknowledge NATO (Grant No. CRG 890461) for financial support.
*Permanent address: Fisica Teorica, Universidad de Sevilla, Apdo, Correos 1065, Sevilla 41080, Spain.

${ }^{1} \mathrm{~J}$. M. Ziman, Principles of the Theory of Solids (Cambridge University Press, London, 1972), Chap. 6.

${ }^{2}$ E. E. Nikitin, Theory of Elementary and Molecular Processes in Gases (Clarendon, Oxford, 1974); E. E. Nikitin and S. Y. Umanskii, Theory of Slow Atomic Collisions (Springer-Verlag, Berlin, 1984).

${ }^{3} \mathrm{M}$. Baer, in Theory of Chemical Reaction Dynamics, edited by M. Baer (CRC, Boca Raton, 1985), Vol. 2, p. 219.

4J. C. Tully, in Modern Theoretical Chemistry, Part B, edited by W. H. Miller (Plenum, New York, 1976), Chap. 5.

5J. Ulstrup, Charge Transfer Processes in Condensed Media (Springer-Verlag, Berlin, 1979).

${ }^{6}$ R. A. Marcus and N. Sutin, Biochim. Biophys. Acta 811, 265 (1985).
${ }^{7}$ Tunneling in Biological Systems, edited by B. Chance, D. C. DeVault, H. Frauenfelder, R. A. Marcus, J. R. Schrieffer, and N. Sutin (Academic, New York, 1979).

${ }^{8}$ D. DeVault, Quantum Mechanical Tunneling in Biological Systems (Cambridge University Press, London, 1984).

${ }^{9}$ K. K. Likharev, Dynamics of Josephson Junctions and Circuits (Gordon and Breach, New York, 1987); K. K. Likharev, IBM J. Res. Dev. 32, 144 (1988).

${ }^{10}$ M. Büttiker, Y. Imry, and R. Landauer, Phys. Lett. 96A, 365 (1983).

${ }^{11}$ S. Washburn and R. A. Webb, Adv. Phys. 35, 375 (1986).

${ }^{12}$ Y. Gefen, E. Ben-Jacob, and A. O. Caldeira, Phys. Rev. B 36, 2270 (1987).

${ }^{13}$ L. Landau, Phys. Z. Sowjetunion 2, 46 (1932).

${ }^{14}$ L. D. Landau and E. M. Lifshitz, Quantum Mechanics (Pergamon, London, 1965), Chap. 7. 
${ }^{15}$ C. Zener, Proc. R. Soc. London Ser. A 137, 696 (1932).

${ }^{16}$ E. C. G. Stueckelberg, Helv. Phys. Acta 5, 369 (1932).

${ }^{17}$ R. Landauer, Phys. Rev. B 33, 6497 (1986).

${ }^{18}$ M. Büttiker, Phys. Rev. B 36, 3548 (1987).

${ }^{19}$ C. P. Slichter, Principles of Magnetic Resonance (Harper \& Row, New York, 1963).

${ }^{20}$ J. D. Macomber, The Dynamics of Spectroscopic Transitions (Wiley, New York, 1976).

${ }^{21}$ K. Mullen, E. Ben-Jacob, Y. Gefen, and Z. Schuss, Phys. Rev. Lett. 62, 2543 (1989).

${ }^{22}$ L. I. Schiff, Quantum Mechanics, 3rd ed. (McGraw-Hill, New York, 1968), p. 289.
${ }^{23}$ G. Baym, Lectures on Quantum Mechanics (Benjamin, New York, 1968), p. 140.

${ }^{24}$ M. S. Child, Discuss. Faraday Soc. 53, 18 (1972).

${ }^{25}$ W. H. Press, B. P. Flannery, S. A. Teukolsky, and W. T. Vettering, Numerical Recipes (Cambridge University Press, Cambridge, England, 1986), Sec. 15.1. We checked the integration scheme by using the adaptive stepsize routine described in Sec. 15.2, where an explicit error tolerance of the integrator is introduced.

${ }^{26}$ R. Landauer and M. Buttiker, Phys. Rev. Lett. 54, 2049 (1985).

${ }^{27}$ J. M. Casado and M. Morillo (unpublished). 